\author{
G. G. Dahlquist • A. Pundziūtẻ-Lyckå • L. Nyström • \\ Swedish Childhood Diabetes Study and the Diabetes \\ Incidence Study in Sweden (DISS) Groups
}

\title{
Birthweight and risk of type 1 diabetes in children and young adults: a population-based register study
}

Received: 28 October 2004 / Accepted: 10 February 2005 / Published online: 30 April 2005

(C) Springer-Verlag 2005

\begin{abstract}
Aims/hypothesis: We investigated the association between type 1 diabetes and birthweight by age at disease onset. Methods: This population-based case-referent study used data from two nationwide case registers that are linked to the Swedish Medical Birth Registry and cover incident cases of type 1 diabetes in the 0 - to 14-year (since 1 July 1977) and 15- to 34-year age groups (since 1 January 1983). Of the cases linked to the Medical Birth Registry, a total of 9,283 cases with onset before 15 years of age was recorded before 1 January 2003, and 1,610 cases were recorded with onset before 30 years of age and born after 1973 (together $95 \%$ of eligible cases). Multiple births and babies of diabetic mothers were excluded. Sexspecific birthweight by gestational week is expressed as multiples of the standard deviation (SDS) and adjusted for year of birth, maternal age and parity. Results: Cases with onset before 10 years of age $(n=5,792)$ showed a significant linear trend in odds ratio (OR) by SDS of adjusted birthweight (OR by SDS: 0.062 ; 95\% CI: 0.037-0.086; $p<0.0001$ ), while cases with onset at the age of 10-29 years showed no significant trend (OR by SDS: $0.004 ; 95 \% \mathrm{CI}$ : -0.007 to $0.0014 ; p=0.22)$. Conclusions/interpretation: The association between type 1 diabetes risk and birthweight seems to be limited to cases with disease onset in younger years.
\end{abstract}

Keywords Birthweight - Children · Intrauterine · Risk factors - Type 1 diabetes

G. G. Dahlquist $(\bowtie) \cdot$ A. Pundziūtè-Lyckå

Department of Clinical Sciences,

Paediatrics, Umeå University,

90185 Umeå, Sweden

e-mail: Gisela.Dahlquist $@$ pediatri.umu.se

Tel.: +46-90-7853876

Fax: $+46-90-123728$

L. Nyström

Department of Public Health and Clinical Medicine, Epidemiology and Public Health Sciences,

Umeå University, Umeå, Sweden
Abbreviations DISS: Diabetes in Sweden Study - OR: odds ratio - SCDS: The Swedish Childhood Diabetes Study - SDS: standard deviation score (denotes the relative position of the individual child relative to the mean birthweight by age and sex expressed as number of standard deviations)

\section{Introduction}

Using population-based prospectively recorded data, we have reported that higher birthweight [1] and more rapid postnatal child growth [2] are risk factors for type 1 diabetes occurring before the age of 15 years. Childhood-onset cases of type 1 diabetes have been rapidly increasing as reported by many countries in Europe [3]. Such a time trend might be due partly to more rapid growth during intrauterine and postnatal periods, as observed in many European countries over the past decades $[4,5]$. The increase in overall growth of children as reflected by increments in height and weight would accelerate an already ongoing autoimmune destruction of beta cells by increasing the need for insulin [6]. Interestingly it has been shown that the incidence of type 1 diabetes is not increasing, but rather decreasing among young adults in Sweden [7]. Therefore the changing incidence over time would reflect an earlier onset rather than a total increase in occurrence of the disease at least up to the age of 35 years.

Using two large population-based prospective diabetes incidence registers and the Swedish Medical Birth Register, we analysed whether the risk for type 1 diabetes is affected by birthweight (adjusted for gestational age) in groups with varying ages of onset of disease.

\section{Subjects and methods}

Subjects and study protocol The study was approved by the research ethics committee at Umeå University and carried out according to the Declaration of Helsinki as 
revised in 2000. Parents and/or diabetic patients consented to be recorded in the registers.

In Sweden all children who are diagnosed with diabetes before the age of 15 years are referred to paediatric departments. Since 1 July 1977 all incident cases of type 1 diabetes are reported to the Swedish Childhood Diabetes Study (SDCS). The completeness of the register varied between 96 and $99 \%$. By 1 January 2003, 9,755 cases (52\% of them males) born in 1973 or later were recorded in this register. Since 1 January 1983, all departments of internal medicine, paediatrics, endocrinology and over 700 primary health care units in Sweden have reported all newly diagnosed cases of diabetes mellitus in the age group 15-34 years to the Diabetes Incidence Study in Sweden (DISS). The reporting doctors classify cases according to clinical criteria as recommended by the World Health Organization. By 1 January 2003, a total of 1,710 cases with type 1 diabetes $(63 \%$ of them males) born in 1973 or later were recorded in the DISS. The completeness of the register varied between 86 and 97\% (mean 91\%) [7]. Only cases classified as type 1 diabetes were included in the present analyses.

The Swedish Medical Birth Registry started in 1973 and contains data on pregnancy, delivery and neonatal period for nearly all infants born in Sweden [5]. Data were obtained from a summary sheet (1973-1982), and since 1983 from copies of the medical documentation from the antenatal care clinics, delivery units and paediatric examination of the newborns.

The three registers were linked using the unique personal identification number given to everyone living in Sweden. Linkage was obtained for a total of 10,893 individuals $(95 \%$ of eligible type 1 diabetic cases): 9,283 from the SCDS and 1,610 from the DISS. Linkage thus failed for 572 individuals, either because the child was born outside Sweden, or there was an error in the identification number used for linkage, or data were missing from the Medical Birth Register (1-2\% of all newborns). All other individuals in the Medical Birth Register were used for comparison adjusting for year of birth, parity, and maternal age. Multiple births, newborns of women diagnosed with diabetes before the pregnancy and individuals who died before 30 years of age were excluded.
We used the following variables from the birth register: sex, birthweight, gestational length, maternal age, parity, maternal delivery and age of diagnosis of diabetes. Birthweights were converted into standard deviation scores (SDS) for sex and gestational age in weeks according to a Swedish standard, based on data from the Medical Birth Register [8]. The score shows the relative position of the individual child in relation to the mean birthweight of newborns of similar sex and gestation, expressed as number of standard deviations. We divided the material into eight SDS classes, from -3 or lower to +3 or higher. Information on sex, birthweight or gestational duration was missing in $3.5 \%$ of individuals. A total of 10,501 individuals with diabetes ( $92 \%$ of those eligible) and 2,888,644 $(51.5 \%$ males) from the non-diabetic population were analysed.

Statistical analyses We used Mantel-Haenszel's technique to estimate the odds ratio (OR) for developing diabetes in a certain weight SDS class, adjusting for year of birth, maternal age (5-year classes), and parity $(1-3 / 4+)$. We determined 95\% CIs using Miettinen's test-based method. Each SDS class was compared with all other SDS classes to avoid the "normality" concept and potential bias with extremes as reference.

In order to estimate linear trends in the odds ratios along the SDS scale, weighted linear regression analyses of the $\log (\mathrm{OR})$ were carried out. The statistical significance of the deviation of the angle coefficient of the regression line from zero was evaluated with a $z$-test, based on the standard error of the angle coefficients. A $p$-value $<0.05$ was considered to be statistically significant.

Three age groups were chosen to reflect the age at which diabetes was diagnosed: $0-9,10-19$ and $20-29$ years. Since the two former groups were much larger $(n=5,792$ and 3,872$)$ than the 20 - to 29 -year group $(n=837)$, the two older age groups were pooled.

\section{Results}

Results for cases with onset before 10 years of age and those with onset between 10 and 29 years are shown in
Table 1 Risk in terms of odds ratios (OR) with $95 \% \mathrm{CI}$, adjusted for year of birth, maternal age and parity for type 1 diabetes diagnosed in the age groups $0-9$ and $10-29$ years by birthweight for gestational age SDS

Reference is all other SDS classes. Only singletons were used in the study and cases of maternal diabetes were excluded

\begin{tabular}{|c|c|c|c|c|c|c|}
\hline \multirow{3}{*}{$\begin{array}{l}\text { Interval of } \\
\text { SDS score }\end{array}$} & \multicolumn{6}{|c|}{ Age at diagnosis of diabetes (years) } \\
\hline & \multicolumn{3}{|l|}{$0-9$} & \multicolumn{3}{|l|}{$10-29$} \\
\hline & Cases $(n)$ & OR & $95 \% \mathrm{CI}$ & Cases $(n)$ & OR & $95 \% \mathrm{CI}$ \\
\hline$<(-3)$ & 16 & 0.71 & $0.44-1.16$ & 14 & 0.65 & $0.39-1.10$ \\
\hline$(-3)$ to $(-2)$ & 119 & 0.88 & $0.73-1.06$ & 123 & 1.01 & $0.84-1.21$ \\
\hline$(-2)$ to $(-1)$ & 644 & 0.83 & $0.76-0.90$ & 641 & 0.96 & $0.88-1.05$ \\
\hline$(-1)$ to 0 & 1,839 & 0.98 & $0.93-1.04$ & 1,470 & 0.94 & $0.88-1.00$ \\
\hline $0-1$ & 1,926 & 1.06 & $1.00-1.12$ & 1,506 & 1.04 & $0.98-1.11$ \\
\hline $1-2$ & 933 & 1.07 & $1.00-1.15$ & 727 & 1.09 & $1.00-1.18$ \\
\hline $2-3$ & 263 & 1.11 & $0.98-1.26$ & 173 & 0.96 & $0.82-1.11$ \\
\hline$>3$ & 52 & 0.94 & $0.69-1.27$ & 55 & 1.13 & $0.82-1.55$ \\
\hline Total $/ p$ for trend & 5,792 & & $<0.0001$ & 4,709 & & 0.22 \\
\hline
\end{tabular}




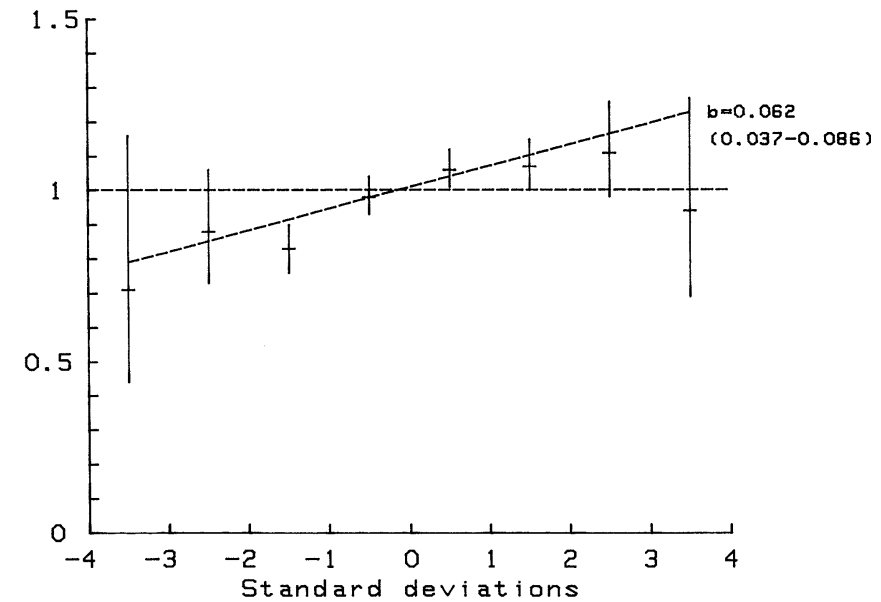

Fig. 1 Odds ratios for the development of diabetes among 0- to 9year-old children plotted against standard deviations in birthweight for gestational duration; 95\% CIs are represented by vertical lines. The angle coefficient of fitted linear regression line (b) and its $95 \%$ CI interval are indicated

Table 1. Figure 1 shows a regression line illustrating the trend for the risk of developing diabetes before the age of 10 years by birthweight SDS. The angle coefficient, denoting the increase in OR for each SDS was $0.062(95 \%$ CI: $0.037-0.086 ; p<0.0001)$. Similar analysis for the age group $10+$ years gave an angle coefficient of $0.004(95 \%$ CI: -0.007 to $0.014 ; p=0.22$ ).

\section{Discussion}

In the present study we confirm previous results, reported by our group and others [1,9], that an increased birthweight is associated with an increased risk of diabetes being diagnosed in childhood. A significant increase in risk by increasing birthweight class was, however, not observed after an age of onset of 10 years or more.

The strengths of the present study are that it is based on prospectively recorded data, uses a large amount of information and includes diabetic cases up to the age of 29 years at diagnosis. We restricted the analyses to singleton births and excluded children born to mothers with prepregnancy diabetes. Adjustments were made for the year of birth, maternal age and parity as recorded in the Medical Birth Register. Routinely registered data may be incomplete, but are unlikely to be systematically biased, as they are recorded long before the child develops diabetes.

A potential source of bias is the difficulty of correctly classifying diabetes into types from the clinical impression obtained at the time of diagnosis in the older age groups. Some individuals with type 2 diabetes may have been misclassified as type 1 diabetics. It is, however, more likely that the true number of type 1 diabetes cases was underestimated, as it is difficult to distinguish cases with type 2 diabetes and latent autoimmune diabetes of adults on the basis of their clinical characteristics. An underestimation of type 1 diabetes would probably not affect our results, while misclassification of type 2 diabetes cases might affect the conclusions due to the association with low birthweight. The proportion of cases with type 2 diabetes is, however, very low in the age group 10-29 years in Sweden, and thus any effect of potential bias should be negligible for the interpretation of our results.

Social status might also be a confounder, since a low birthweight is more prevalent among mothers with lower social status. On the other hand, we have previously shown [10] that in a Swedish setting, determinants of low social status of the parents are associated with an increased risk of childhood diabetes, thus leading to the reverse effect.

Birthweight [5], as well as the prevalence of overweight children [7], has gradually increased in Sweden over recent decades. This trend seems to parallel the increasing incidence of type 1 diabetes occurring in childhood, especially in the younger age-at-onset groups, but is not seen in age groups older than 15 years at diagnosis [7]. One explanation could be that the overload of the beta cells due to increased insulin demand in the growing child may accelerate the process of beta-cell destruction and lead to an earlier clinical diagnosis of diabetes. The effect of birthweight alone may persist only for the first decade of life.

In conclusion, the importance of a high birthweight as a risk factor for type 1 diabetes seems to be limited to youngonset cases. The increase in incidence of type 1 diabetes seen in Sweden in age groups younger than 10 years, but not in young adults, might be partly explained by the increasing birthweight.

Acknowledgements This project was supported by grants from the Swedish Research Council (project number 07531), the Swedish Diabetes Association and the Västerbotten County Council. We also wish to thank the National Board of Health and Welfare for providing datasets and for expert help with the analyses.

\section{Members of the Swedish Childhood Diabetes Study}

Lars Skogsberg (Boden), Agne Lindh (Borås), Karin Segnestam (Eskilstuna), Kalle Snellman (Falun), Åke Stenberg and Christer Nilsson (Gällivare), Gunilla KördelEngberg (Gävle), Bengt Lindblad (Göteborg), Nils Östen Nilsson (Halmstad), Jan Neiderud (Helsingborg), Herje Hörnell and Åke Lagervall (Hudiksvall), Lars Ivar Hardell (Kalmar), Hans Edenwall (Karlskrona), Gudrun Jonsell (Karlstad), Karin Larsson (Kristianstad), Bengt Hansing (Lidköping), Sture Sjöblad (Lund), Lars Stenhammar (Norrköping), Lennart Hellenberg (Nyköping), Elena Lundgren (Skellefteå), Marie Bourdin (Skövde), Britta Björsell (Sollefteå), Torunn Torbjörnsdotter (Huddinge), Susanne Rudberg/Ingemar Zachrisson (Stockholm), Eva Landgren (Sundsvall), Ragnar Hanås (Uddevalla), Jan Gustafsson (Uppsala), Margareta Blomgren (Visby), Nils Wramner (Trollhättan), Björn Eriksson (Västervik), Björn Jönsson (Ystad), Carl Göran Arvidsson (Västerås), Stig Edvardsson (Växjö), Ulf Ståhle (Ängelholm), Jan Åman (Örebro), Stellan Sjögren (Örnsköldsvik), Anna-Lena Nilsson (Östersund) 


\section{Members of the Diabetes in Sweden Study Group}

Hans Arnqvist (Linköping), Annika Ekbom-Schnell (Uppsala), Soffia Gudbjornsdottir (Gothenburg), Jan Bolinder (Stockholm), Jan Eriksson (Umeå), Göran Sundkvist (Malmö)

\section{References}

1. Dahlquist G, Bennich SS, Kallen B (1996) Intrauterine growth pattern and risk of childhood onset insulin dependent (type I) diabetes: population based case-control study. BMJ 313:11741177

2. Blom L, Persson LA, Dahlquist G (1992) A high linear growth is associated with an increased risk of childhood diabetes mellitus. Diabetologia 35:528-533

3. Green A, Patterson CC (2001) Trends in the incidence of childhood-onset diabetes in Europe 1989-1998. Diabetologia 44 [Suppl 3]:B3-B8

4. Livingstone B (2000) Epidemiology of childhood obesity in Europe. Eur J Pediatr 159 [Suppl 1]:S14-S34
5. Odlind V, Haglund B, Pakkanen M, Otterblad Olausson P (2003) Deliveries, mothers and newborn infants in Sweden, 1973-2000: trends in obstetrics as reported to the Swedish Medical Birth Register. Acta Obstet Gynecol Scand 82:516528

6. Dahlquist G (1995) Environmental risk factors in human type 1 diabetes - an epidemiological perspective. Diabetes Metab Rev $11: 37-46$

7. Pundziute-Lycka A, Dahlquist G, Nystrom L et al (2002) The incidence of type 1 diabetes has not increased but shifted to a younger age at diagnosis in the $0-34$ years group in Sweden 1983 to 1998 . Diabetologia 45:783-791

8. Källen B (1995) A birth weight for gestational age standard based on data in the Swedish Medical Birth Registry, 19851989. Eur J Epidemiol 11:601-606

9. Stene LC, Magnus P, Lie RT, Sovik O, Joner G (2001) Birth weight and childhood onset type 1 diabetes: population based cohort study. BMJ 322:889-892

10. Blom L, Dahlquist G, Nyström L, Sandström A, Wall S (1989) The Swedish Childhood Diabetes Study - social and perinatal determinants for diabetes in childhood. Diabetologia 32:7-13 